\title{
Photoionized nebulae in the Local Group: Nucleosynthesis and chemical evolution
}

\author{
Walter J. Maciel ${ }^{* \dagger}$ \\ University of São Paulo \\ E-mail: wjmaciel@iag.usp.br
}

Roberto D. D. Costa

University of São Paulo

E-mail: roberto.costa@iag.usp.br

\section{Oscar Cavichia}

Federal University of Itajubá

E-mail: cavichia@unifei.edu.br

\begin{abstract}
Photoionized nebulae, comprising HII regions and planetary nebulae (PN), are excellent laboratories to study the nucleosynthesis and chemical evolution of several elements in the Galaxy and in other galaxies. While HII regions reflect basically the present interstellar abundances of elements such as oxygen, sulphur, neon, etc., planetary nebulae display the original composition at the time their progenitor stars were formed. On the other hand, the abundances of elements such as $\mathrm{He}$ and $\mathrm{N}$ are affected by the evolution of the PN progenitor stars, so that a direct comparison of the abundances of these elements in photoionized nebulae gives important clues on the chemical evolution processes of their host galaxies.

There is presently a very large quantity of abundance data on photoionized nebula for several galaxies in the Local Group, both regarding HII regions and PN. In particular, our group has obtained one of the largest samples of accurately measured abundances of PN in several regions of the Milky Way and the Magellanic Clouds, which can be supplemented by data from other surveys of Local Group galaxies in order to derive a statistically significant sample.

In this work we investigate the main distance-independent correlations involving the elements He, $\mathrm{N}, \mathrm{O}, \mathrm{Ne}, \mathrm{S}$, and Ar in photoionized nebulae located in Local Group galaxies. It is shown that most of these correlations hold for all the objects in the Local Group, indicating that similar chemical evolution processes have occurred in these objects. These correlations can also be compared with predictions of theoretical models both for intermediate and large mass stars, so that some implications can be derived for the nucleosynthesis processes in these stars.
\end{abstract}

XIII Nuclei in the Cosmos,

7-11 July, 2014

Debrecen, Hungary

*Speaker.

${ }^{\dagger}$ Work partially supported by FAPESP/CNPq. 


\section{Introduction}

Abundances of some heavy elements such as $\mathrm{O}, \mathrm{Ne}, \mathrm{S}$, and $\mathrm{Ar}$ in planetary nebulae are not expected to be affected in a signficant way by the evolution of their progenitor stars. In fact, the average abundances of these elements are not very different from the observed abundances in HII regions, taking into account the age-metallicity relation in the Galaxy and the existence of radial abundance gradients. Therefore, the measured abundances of these elements in PN reflect the interstellar abundances at the time the progenitor stars were formed, and can be compared with the abundances of red giants, AGB stars, and younger objects, such as HII regions and Blue Compact Galaxies (BCG). On the other hand, the abundances of elements such as $\mathrm{He}, \mathrm{N}$, and $\mathrm{C}$ are affected by the evolution of the PN progenitor stars, so that an analysis of these abundances can be used to test nucleosynthesis predictions of theoretical models for intermediate mass stars.

New data on the chemical abundances of planetary nebulae in different systems have been obtained by our group, (see for example Idiart et al. [15], Maciel et al. [22], [23], Cavichia et al. [7], [8]). These systems include the solar neighbourhood, the galactic disk, the galactic bulge and the Magellanic Clouds. Abundances of the main chemical elements have been determined, namely, $\mathrm{He}, \mathrm{N}, \mathrm{O}, \mathrm{Ne}, \mathrm{S}$ and Ar, with average uncertainties in the $0.2-0.3$ dex range. In this work, we consider the abundances of these elements in PN and compare these results with abundance data for HII regions and BCG both in the Galaxy and in other objects of the Local Group.

\section{The data}

This work is based on a very large sample of PN and HII regions, both in the Galaxy and in other objects of the Local Group. We have included about 400 PN in the Galaxy, 500 PN in the Magellanic Clouds, and about 200 PN in other galaxies. For HII regions, the samples are smaller, comprising 200 objects in the Galaxy, and about 300 elsewhere. Apart from our own data, we have considered PN data from the following sources: Girard et al. [10]; Henry and collaborators [13], [12], [30]; Milanova and Kholtygin [29]; Stasińska et al. [39], Leisy and Dennefeld [21]; Chiappini et al. [9] [Milky Way, Magellanic Clouds]. In agreement with some conclusions by Henry et al. [13], part of the results by Leisy and Dennefeld [21] are overestimates, and have not been considered here. In this sense, our results are an update and improvement over the results by Stasińska et al. [39], where an attempt was made to compare the PN population in five galaxies; Kwitter et al. [20]; Balick et al. [2] [M31]; Richer and McCall [33] [M32, NGC 185, NGC 205]; Bresolin et al. [5]; Magrini et al. [26] [M33]; Stasińska et al. [38] [NGC 300]; Peña et al. [31] [NGC 3109]; Magrini et al. [25] [Sextans A, B].

For HII regions and BCG the data are from Afflerbach et al. [1]; Guseva et al. [11]; Rudolph et al. [36]; Tsamis et al. [40] [MW, MC]; Izotov and Thuan [17]; Izotov et al. [16] [BCG]; Zurita and Bresolin [41]; Sanders et al. [37]; Carigi and Peimbert [6] [M31]; Bresolin et al. [5]; Magrini et al. [27]; Rosolowsky and Simon [35] [M33]; Bresolin et al. [3] [M51]; Stasińska et al. [38]; Bresolin et al. [4] [NGC 300]; Peña et al. [31] [NGC 3109]; Kennicutt et al. [19]; Carigi and Peimbert [6] [M101]; Magrini et al. [25] [Sextans A, B]. 

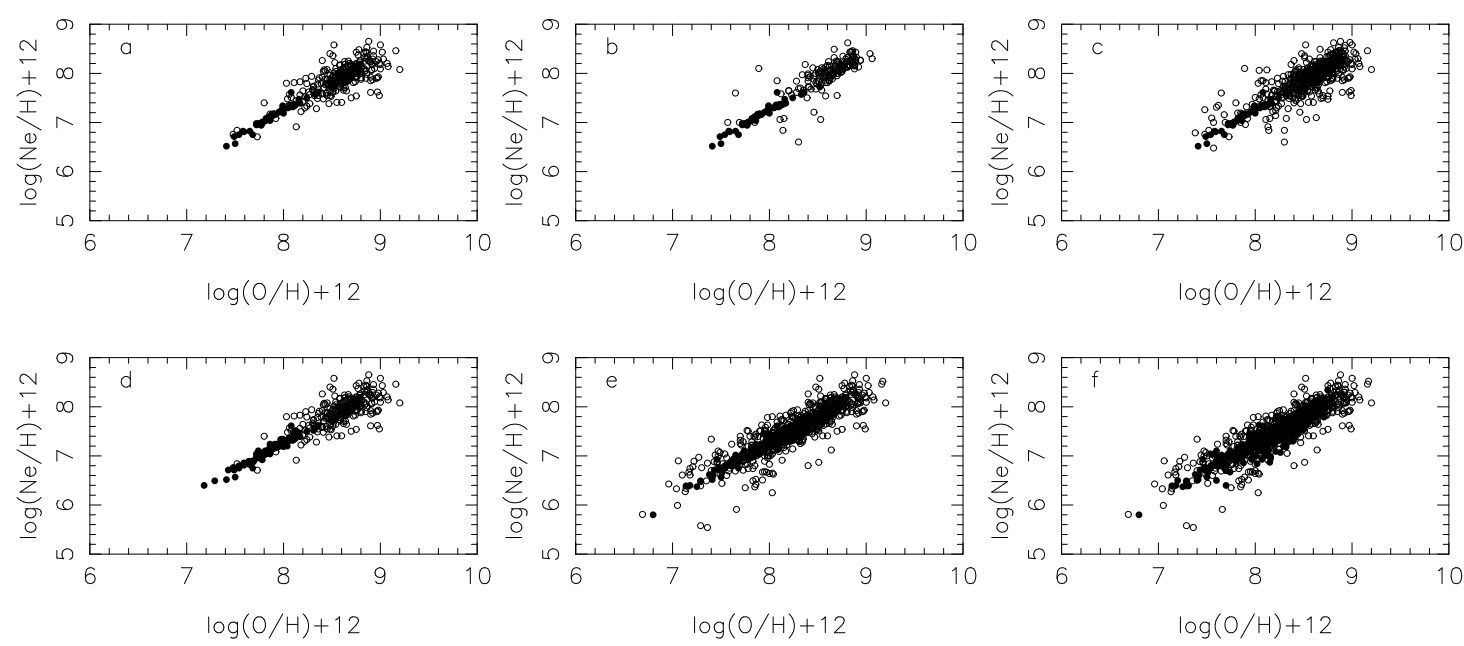

Figure 1: $\mathrm{Ne} / \mathrm{H} \times \mathrm{O} / \mathrm{H}$ for different combination of samples. $\mathrm{PN}$ (empty circles), HII regions (filled circles).

\section{Elements not produced by the progenitor stars}

In view of the large uncertainties in the PN distances, it is interesting to investigate some distance-independent correlations, such as the relations of $\mathrm{Ne} / \mathrm{H}, \mathrm{S} / \mathrm{H}$, and $\mathrm{Ar} / \mathrm{H}$ as functions of the oxygen abundance $\mathrm{O} / \mathrm{H}$, which acts as a proxy for the metallicity, being correlated with the iron abundance $[\mathrm{Fe} / \mathrm{H}]$ (see for example Ramirez et al. [32]). These abundances generally vary approximately in lockstep with oxygen, as predicted by stellar nucleosynthesis theory. In the following we will discuss in detail the Ne data, which show the better results, altough for $\mathrm{S} / \mathrm{H}$ and $\mathrm{Ar} / \mathrm{H}$ similar results are obtained.

The variation of the $\mathrm{Ne} / \mathrm{H}$ ratio with $\mathrm{O} / \mathrm{H}$ is shown in Figure 1a for Milky Way PN (empty circles) and HII regions (filled cicles). It shows a very good correlation implying an essentially constant Ne/O ratio. The PN dispersion is higher than in the case of HII regions, since PN are older objects and any given sample probably includes objects of different ages, as we have concluded elsewhere (Maciel et al. [24]). It can also be seen that the $\mathrm{O} / \mathrm{H}$ range is similar both for PN and HII regions, which essentially reflects the fact that the interstellar metallicities did not change appreciably in the last $5 \mathrm{Gyr}$ approximately, a result that is supported by recent determinations of the age-metallicity in the Milky Way (see for example Rocha-Pinto et al. [34]). A comparison with the more homogeneus results from Henry and collaborators is shown in Figure 1b. The results are similar, in the sense that both PN and HII define essentially the same correlation, but the average dispersion of the PN data is somewhat smaller for the Henry et al. data, as expected. On the other hand, some PN at the lower left corner in their sample seem to have a higher dispersion than the other objects.

We can have an idea of the increase in the dispersion by considering all PN data for the Milky Way as shown in Figure 1c. It confirms that the correlation is maintained at approximately the same dispersion. The inclusion of BCG does not change this results, as can be seen from Figure 1d. These objects concentrate towards lower metallicities, $\varepsilon(\mathrm{O})=\log (\mathrm{O} / \mathrm{H})+12<8.2$. The inclusion of the emission line galaxies (ELG) from Izotov et al. [16] does not change this result.

Including now objects in the LMC and SMC we have Figure 1e, which again includes BCG 
galaxies. The $\mathrm{Ne} / \mathrm{H}$ correlation is the same, but a clear enhancement occurs for lower metallicities, which clearly reflects the fact that the Magellanic Clouds are more metal-poor than the Galaxy. Apart from a few points at very low metallicities $(\varepsilon(O)<7.7)$ the same dispersion is observed at all metallicities, amounting to about 0.4 to $0.5 \mathrm{dex}$, higher than the individual abundance uncertainties, so that it is probably real, reflecting the different ages of the PN central stars. Finally, Figure if shows the results for $\mathrm{Ne} / \mathrm{H}$ for a larger sample including all objects in the Local Group galaxies. It is amazing how these different objects present the same correlation as in the Milky Way, that is, despite their different metallicities and morphologies, their nucleosynthetic processes and chemical evolution apparently follow the same rules. It is also interesting to notice that in this larger sample the observed range of oxygen and in neon abundances are similar for PN and HII regions.

From Figure 1 (especially 1f) it can be seen that the dispersion of the PN data is approximately constant for all metallicities, so that any Ne contribution from the progenitor stars is probably smaller or similar to the average uncertainties. For HII regions, it seems to increase with the metallicity, which may be an effect of larger samples and of the age-metallicity relation.

For argon the results are similar compared to neon, but the dispersion is higher. The comparison with HII regions suffers from the lack of data for this element. The analysis of all PN data for the Milky Way maintains these characteristics. The inclusion of BCG and ELG clearly improves the correlation, showing that the correlation defined at higher metallicities still hold for lower oxygen abundances, namely $\varepsilon(\mathrm{O})<8.2$. The same can be said of the inclusion of the Magellanic Clouds. Although the $\mathrm{Ar} / \mathrm{H}$ dispersion is higher for $\mathrm{PN}$, in the case of $\mathrm{HII}$ regions it is essentially the same as for $\mathrm{Ne} / \mathrm{H}$, so that the $\mathrm{HII}$ regions are more homogeneous than the $\mathrm{PN}$, which reflects their very low ages, roughly a few million years. Clearly, within such a short time bracket the average interstellar abundances are not expected to change appreciably. Finally, considering the remaining objects of the Local Group, we have a similar result. In this case, even the HII regions present a slightly higher dispersion, which reflects the different metallicities of the Local Group objects, but the dispersion in the PN data is considerably higher.

For sulphur a good correlation is observed for HII regions in the Milky Way, but the galactic PN display what is usually called the "sulphur anomaly", that is, many PN apparently have somewhat lower S/H abundances for their metallicity (see for example Henry et al. [12], [14]). The sulphur anomaly has been attributed to a deficiency in the calculation of the sulphur ICFs, lack of accurate atomic constants, effect of the nucleosynthesis in the progenitor stars, and grain formation. This effect is even more pronounced in the samples by Henry and collaborators, where very few objects seem to lie above the curve defined by the HII regions. The inclusion of all galactic PN data leads to a more even distribution, and the sulphur anomaly becomes less important. The inclusion of BCG and ELG maintains these conclusions, that is, the sulphur abundances of HII apparently do not suffer from the sulphur anomaly, which seems to be a characteristic of the empirical determination of sulphur abundances in PN. In other words, the problem of the sulphur determinations affecs basically the planetary nebulae, but not the HII regions and BCG.

\section{Elements produced by the progenitor stars}

The main elements that are produced by the $\mathrm{PN}$ progenitor stars are $\mathrm{He}$ and $\mathrm{N}$, for which there are generally good quality data for PN and to a lesser extent, for HII regions. Carbon is also an 


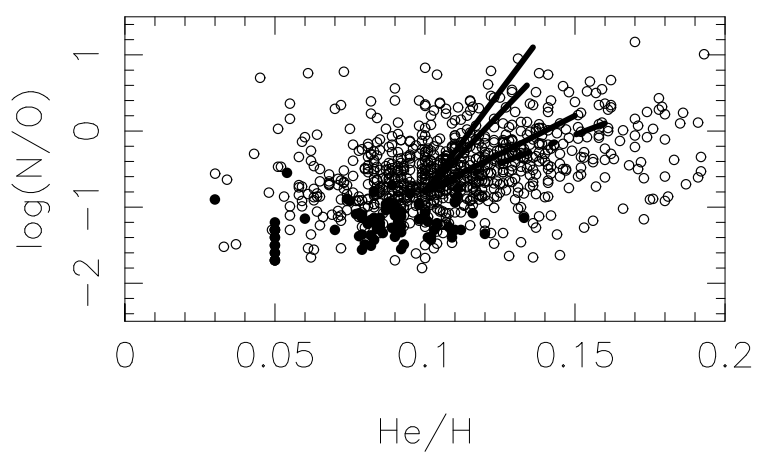

Figure 2: $\mathrm{N} / \mathrm{O} \times \mathrm{He} / \mathrm{H}$ for PN and $\mathrm{HII}$ regions and models by Karakas (solid) and Marigo (dashed).

interesting element, but there are very few objects with accurate carbon abundances, so this element will not be considered in this work. Also, He determinations in HII regions are frequently affected by the presence of neutral helium, so that in this case the derived abundances are usually lower limits. There is a general trend for $\mathrm{N} / \mathrm{H}$ and N/O to increase as $\mathrm{He} / \mathrm{H}$ increases for galactic PN, but the dispersion is higher than in the case of the previous elements, which clearly reflects the fact that the nitrogen abundances measured in PN include both the pristine nitrogen plus the contribution from the dredge up processes in the red giant progenitors. $\mathrm{N} / \mathrm{H}$ is well correlated with $\mathrm{O} / \mathrm{H}$, but again at a larger dispersion, and the PN are frequently located above the average curve defined by the HII regions, again confirming the excess $\mathrm{N}$ in PN. The inclusion of the whole galactic PN sample increases the dispersion, but the general trend with $\mathrm{O} / \mathrm{H}$ is maintained. The inclusion of the BCG and ELG clearly shows the lower N/H abundances in these objects. The Magellanic Cloud PN also follow the same trends, and display larger N/H abundances compared with HII regions, and including all Local Group objects the dispersion is even larger, especially for PN. The same tendency of the $\mathrm{Ne} / \mathrm{H}$ data is maintained for the relation between $\mathrm{N} / \mathrm{H}$ and $\mathrm{O} / \mathrm{H}$, including the $\mathrm{N}$ excess of PN compared with HII regions. For these objects the dispersion is smaller than for PN, but greater than in the case of the lower metallicity BCG. A comparison of the N/O abundances with predictions of theoretical models for intermediate stars in the mass range of 1 to $5 \mathrm{M}$. can be seen in Figure 2, where we included theoretical models by Marigo et al [28] ( $Z=0.019$, dashed line) and Karakas [18] $(Z=0.02,0.004,0.008$, solid lines).

Acknowledgements. This work was partially supported by FAPESP and CNPq.

\section{References}

[1] Afflerbach, A., Churchwell, E., Werner, M. W. 1997, ApJ 478, 190

[2] Balick, B., Kwitter, K. B., Corradi, R. L. M., Henry, R. B. C. 2013, ApJ 774, 3

[3] Bresolin, F., Garnett, D. R., Kennicutt, R. C. 2004, ApJ 615, 228

[4] Bresolin, F., Gieren, W., Kudritzki, R. P. et al. 2009, ApJ 700, 309

[5] Bresolin, F., Stasińska, G., Vilchez, J. M. et al. 2010, MNRAS 404, 1679

[6] Carigi, L., Peimbert, M. 2011, RMAA 47, 139

[7] Cavichia, O., Costa, R. D. D., Maciel, W. J. 2010, RMAA 46, 159 
[8] Cavichia, O., Costa, R. D. D., Maciel, W. J. 2011, RMAA 47, 49

[9] Chiappini, C., Gorny, S. K., Stasińska, G., Barbuy, B. 2009, A\&A 494, 591

[10] Girard, P., Köppen, J., Acker, A. 2007, A\&A 463, 265

[11] Guseva, N. G., Izotov, Y. I., Papaderos, P., Fricke, K. J. 2007, A\&A 464, 885

[12] Henry, R. B. C., Kwitter, K. B., Balick, B. 2004, AJ 127, 2284

[13] Henry, R. B. C., Kwitter, K. B., Jaskot, A. E. et al. 2010, ApJ 724, 748

[14] Henry, R. B. C., Speck, A., Karakas, A. et al. 2012, ApJ 749, 61

[15] Idiart, T. E. P., Maciel, W. J., Costa, R. D. D. 2007, A\&A 472, 101

[16] Izotov, Y. I., Stasińska, G., Meynet, G. et al. 2006, A\&A 448, 955

[17] Izotov, Y. I., Thuan, T. X. 1999, ApJ 511, 639

[18] Karakas, A. 2010, MNRAS 403, 1413

[19] Kennicutt, R. C., Bresolin, F., Garnett, D. R. 2003, ApJ 591, 801

[20] Kwitter, K. B., Lehman, E. M. M., Balick, B., Henry, R. B. C. 2012, ApJ 753, 12

[21] Leisy, P., Dennefeld, M. 2006, A\&A 456, 451

[22] Maciel, W. J., Costa, R. D. D., Idiart, T. E. P. 2009, RMAA 45, 127

[23] Maciel, W. J., Costa, R. D. D., Idiart, T. E. P. 2010, Nuclei in the Cosmos XI, PoS

[24] Maciel, W. J., Costa, R. D. D., Idiart, T. E. P. 2010, A\&A 512, A19

[25] Magrini, L., Leisy, P., Corradi, R. L. M. et al. 2005, A\&A 443, 114

[26] Magrini, L., Stanghellini, L., Villaver, E. 2009, ApJ 696, 729

[27] Magrini, L., Vilchez, J., Mampaso, A. et al. 2007, A\&A 470, 865

[28] Marigo, P., Bernard-Salas, J. et al. 2003, A\&A 409, 619

[29] Milanova, Yu. V., Kholtygin, A. F. 2009, Astron. Lett. 35, 518

[30] Milingo, J. B., Kwitter, K. B., Henry, R. B. C., Souza, S. P. 2010, ApJ 711, 619

[31] Peña, M., Stasińska, G., Richer, M. G. 2007, A\&A, 476, 745

[32] Ramirez, I., Allende-Prieto, C., Lambert, D. L. 2013, ApJ 764, 78

[33] Richer, M. G., McCall, M. L. 2008, ApJ 684, 1190

[34] Rocha-PInto, H. J., Maciel, W. J., Scalo, J., Flynn, C. 2000, A\&A 358, 850

[35] Rosolowsky, E., Simon, J. D. 2008, ApJ 675, 1213

[36] Rudolph, A. L., Fich, M., Bell, G. R. 2006, ApJS 162, 346

[37] Sanders, N. E., Caldwell, N., McDowell, J., Harding, P. 2012, ApJ 758, 133

[38] Stasińska, G., Peña, M., Bresolin, F., Tsamis, Y. G. 2013, A\&A 552, A12

[39] Stasińska, G., Richer, M. G., McCall, M. L. 1998, A\&A 336, 667

[40] Tsamis, Y. G., Barlow, M. .J., Liu, X.-W. 2003, MNRAS 338, 687

[41] Zurita, A., Bresolin, F. 2012, MNRAS 427, 1463 